\title{
Telerobotic Mini-Golf: Interactive Learning Performance of Human Teleoperators
}

\author{
Sudath R. Munasinghe ${ }^{1}$, Ju-Jang Lee ${ }^{1}$, Tatsumi Usui ${ }^{2}$, Masatoshi Nakamura ${ }^{2}$, Naruto Egashira ${ }^{3}$ \\ ${ }^{1}$ Dept. of Electrical Engineering and Computer Science, Korea Advanced Institute of Science and \\ Technology, 373-1 Guseong-dong, Yuseong-gu, Dejeon 305-701, Korea \\ rohan@odyssey.kaist.ac.kr \\ ${ }^{2}$ Dept. of Advanced Systems Control Engineering, Saga University, 1 Honjomachi, \\ Saga 840-8502, Japan \\ usui@cntl.ee.saga-u.ac.jp \\ ${ }^{3}$ Dept. of Control and Information Systems Engineering, Kurume National College of \\ Technology 1-1-1 Kumorino Kurume-City Fukuoka 830-8555, Japan \\ naruto@kurume-nct.ac.jp
}

\begin{abstract}
A telerobotic test-bed has recently been built between KAIST(Korea) and Saga University(Japan). This paper presents its first research work on investigating the interactive learning performance of human teleoperators. For this purpose, the telerobotic test-bed was re-shaped to a telerobotic golf system. Eleven players were participated, and their interactive performance with the telerobot was carefully analyzed for the errors and skills that they demonstrated. It was observed that human teleoperators first try to avoid errors that they did in the previous trials, and then make efforts to explore new skills. It was also experienced that simple task oriented design features and their coordination could significantly improve telerobotic system performance without making it to an excessively sophisticated shape. Interactive learning with naked-eye vision was compared with that of camera vision, and an effective training procedure for human teleoperators was revealed. Some simple, yet appropriate techniques have also been identified that significantly enhance the performance of human teleoperators.
\end{abstract}

Keywords: Teleoperation, Interactive learning, Telerobotic mini-golf

\section{INTRODUCTION}

Telerobotics is becoming an increasingly important research and application area. Telerobotics and teleoperations have been introduced in a wide range of applications recently. Ranging from space and onorbit [1], teleoperations have found its potential in undersea [2], medical [3], welfare [4], rescue [5], and entertainment [6] applications. The trend of telerobotics has been stimulated with its Internetbased implementation [7], [8]. The classical problems of telerobotics are:

(a) Instability caused by time delay

(b) Poor visual feedback (with 2D cameras)

Time delay instability has been thoroughly analyzed in [9], [10], and proposed the supervisory control that ensures stable teleoperation. For the visual feedback problem, virtual reality based techniques have been suggested [11], [12], [13]. Graphics-intensive systems generally consume a significant time for vision data processing. When object recognition [14] and modeling are required, such systems render additional errors to the operator interface, thereby, degrading effective operation.
Although efforts are taken to develop sophisticated systems, it seems that there has not been a thorough investigation to understand and describe how the human operator cognitively interact with the telerobot [15], and his learning and adaptation patterns. Such information might provide very important clues to develop telerobotic systems that are capable of exploiting teleoperator skills by way of properly coordinated, simple, yet appropriate techniques. This paper is aimed along this direction of research, where we are particularly interested in thoroughly observing the interactive learning performance of human teleoperators. The recently built telerobotic test-bed between KAIST(Korea) and Saga University(Japan) was reshaped to a simple, yet challenging telerobotic golf system. Eleven players have been involved in playing the game producing valuable data and experience. By analyzing their interactive actions, and what they experienced, some key issues in telerobotics have been observed with more clarity. Teleoperation with nakedeye vision has been found to be an effective pre-training for teleoperators before they actually interact with camera vision. Simple, yet effective developments have also been identified. 


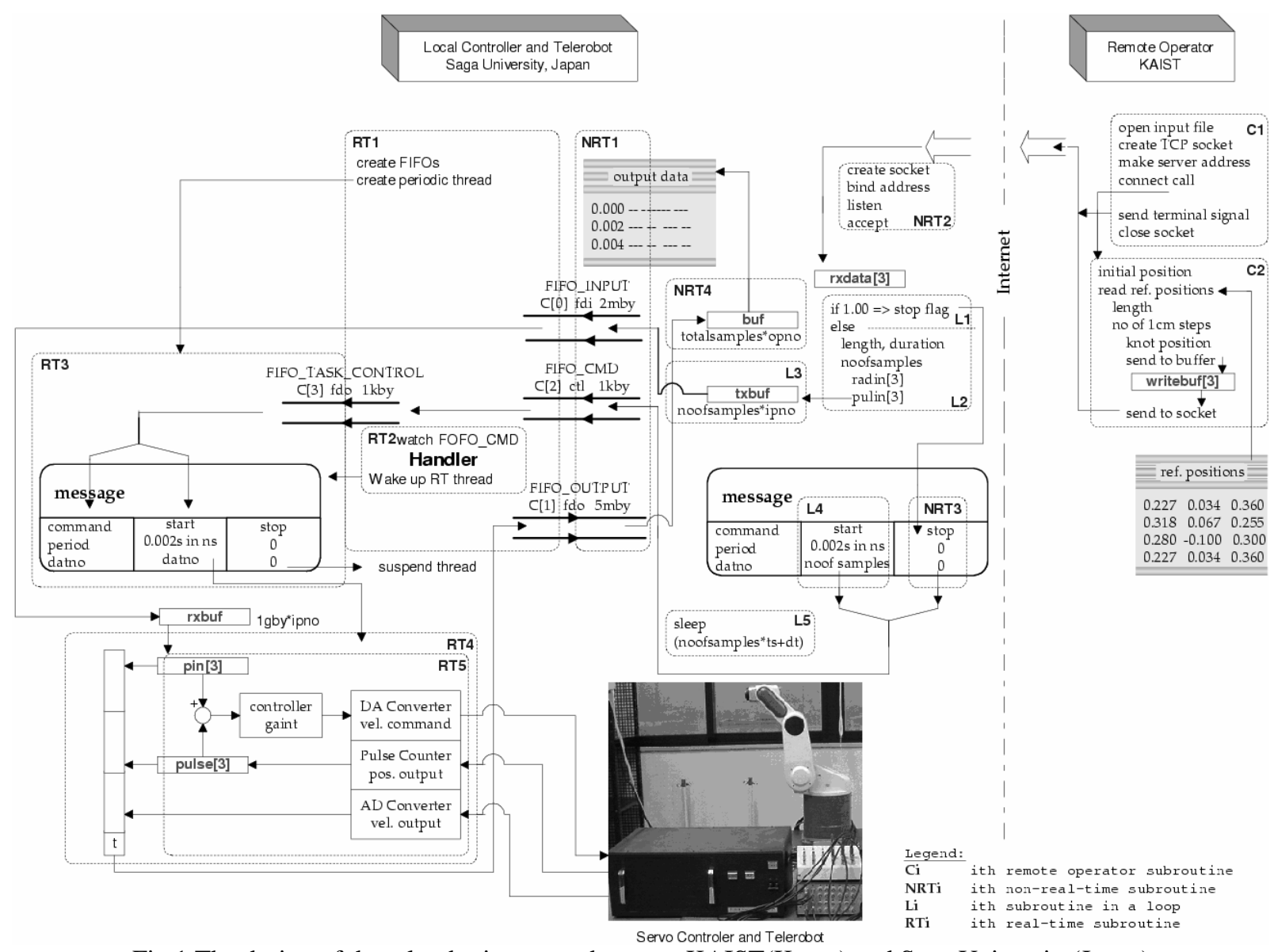

Fig.1 The design of the telerobotic system between KAIST(Korea) and Saga University(Japan)

\section{THE TELEROBOTIC TESTBED}

\subsection{Design Features}

Figure 1 illustrates the recently built telerobotic testbed between KAIST(Korea) and Saga University(Japan). The remote operator terminal resides in KAIST, and the local controller and telerobot reside in Saga University(Japan) as illustrated in Fig. 1. The two sides communicate through TCP sockets over the Internet. Remote operator terminal reads motion commands from a data file, or through the keyboard, and sends those commands to the local controller in Saga University (C1). The local controller runs a non real-time loop (L1-L4) within which it reads the incremental positions, and calculates distance between them, duration for the incremental motion, and number of input samples in each incremental motion (L1 and L2). The input data for the incremental motion of the telerobot is sent to a serial buffer and then placed onto FIFO $\_$INPUT (L3). This incremental motion is planned assuming uniform end-effector speed. Then, the "start" command is written onto the "message" structure, together with the number of input data samples for the incremental motion (L4). The real-time control loop (RT4) executes the feedback control of the robot while reading motion data from the FIFO $\backslash$ INPUT. When there is no motion data in FIFO $\backslash$ INPUT (temporarily), then the RT5 control loop operates. RT5 uses the last position data and stabilizes the robot at that joint configuration until next incremental position data appears on FIFO \_INPUT. However, RT5 does not write position output (which is unnecessary) to the FIFO $\_O U T P U T$.

\subsection{Task oriented System Design for Telerobotic Golf}

By way of task oriented design, the telerobotic test-bed was customized to a telerobotic golf system as shown in Fig. 2(a). The key design features are explained as follows: 


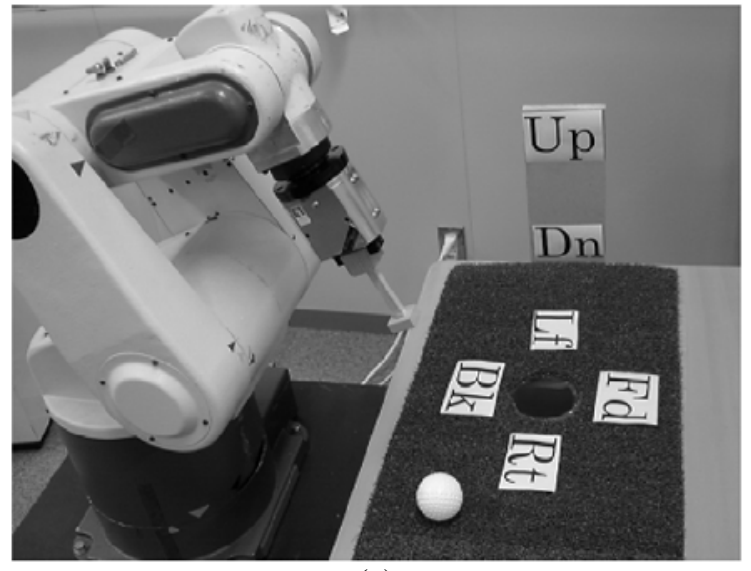

(a)

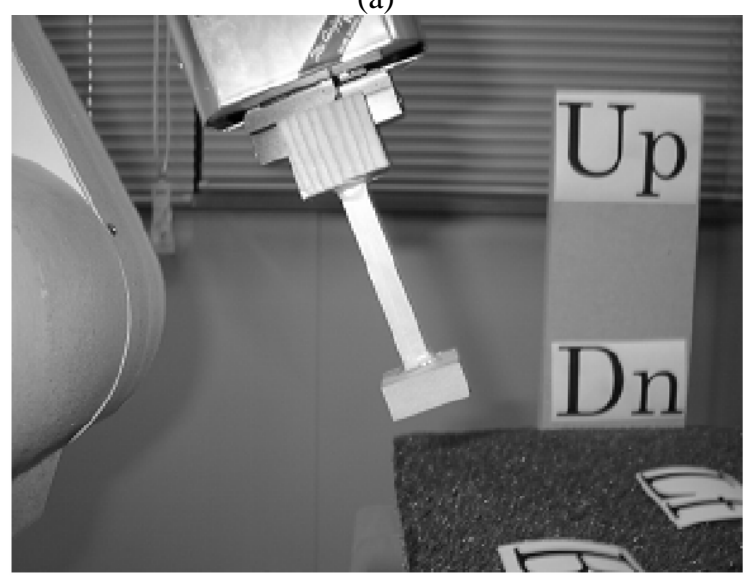

(b)

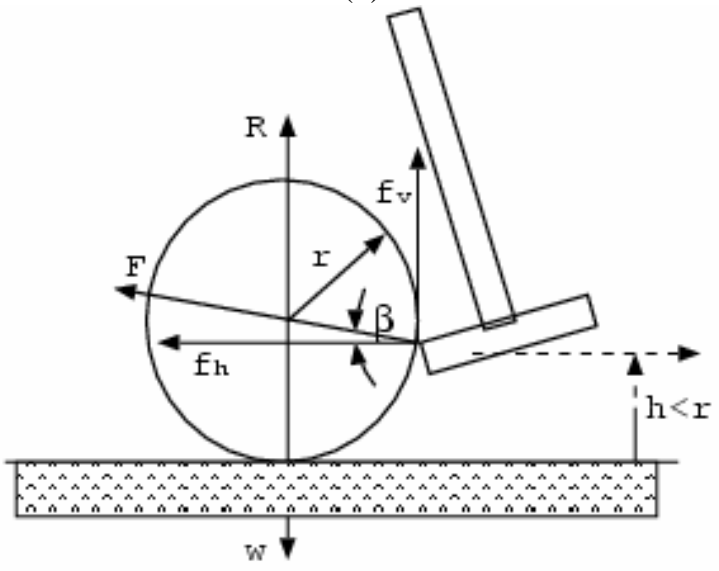

(c)

Fig.2 Telerobotic golf: (a) The golf court, (b) Putter design, and (c) Force diagram of the putting stroke

\section{Putter Design}

The putter was designed as shown in Fig. 2(b), with a thin long handle terminating at $1[\mathrm{~cm}]$ thick, square shaped piece of wood. The handle is sufficiently thin so that it does not block the view of the ball and the hole during the play. The dimensions of the square shaped end are sizable so that its positioning is not required to be precise before putting the ball. The thickness of the tool is small enough so that it always contacts the ball close to the ground, thereby, avoiding unnecessary force on the ground. This feature also provides more rolling torque, which facilitates motion. Figure 2(c) shows the forces acting on the ball when it is in contact with the putter, which is held closer to the ground. The rolling torque $\tau=F r \cos \beta$, where $F$ is the contact force on the ball, $r$ is the radius of the ball, and $\beta=\sin ^{-1}(r-h) / r ; h<r$ being the height of the contact point from the ground. If the condition $h<r$ is satisfied, the reaction force at the contact point of the ball and the ground is $R=w-f_{v}$, where $w$ is the weight of the ball and $f_{v}=F \sin \beta$ is the vertical component of $F$. This condition helps ball movement along the ground.

\section{Control System Design}

The industrial manipulator shown in Fig. 2(a) has five degrees of freedom in R-R-R-R-P configuration. The first joint has a vertical axis of rotation, whereas next three joints have horizontal axes of rotation. In the telerobotic golf design, only the first three joints were used. All three joints are rate controlled in joint coordinates $\left(\theta_{1}, \theta_{2}, \theta_{3}\right)$ in response to a motion command in Cartesian co-ordinates $\left(x, y^{-} z\right)$. The inverse kinematic transformation is given by

$$
\begin{aligned}
& \theta_{1}=\tan ^{-1}(y / x) \\
& \theta_{2}=\tan ^{-1}(c / z)-\cos ^{-1} \frac{L_{2}^{2}-L_{3}^{2}+c^{2}+z^{2}}{2 L_{2} \sqrt{c^{2}+z^{2}}} \\
& \theta_{3}=\pi-\cos ^{-1}\left(L_{2}^{2}+L_{3}^{2}-c^{2}-z^{2}\right) /\left(2 L_{2} L_{3}\right)
\end{aligned}
$$

where ${ }^{c}=\sqrt{x^{2}+y^{2}}-L_{1}$, and $L j$ is the length of $j$ th link. Independent servo actuators implement the feedback control law $u^{j}=K_{v}\left\{K p\left(\theta_{i n}^{j}-\theta_{\text {out }}^{j}\right)-\dot{\theta}_{\text {out }}^{j}\right\}$, where $\theta_{\text {in }}^{j}, \theta_{\text {out }}^{j}, \dot{\theta}_{\text {out }}^{j}$ are position input, position output, and velocity output of $j$ th joint. Parameters $K_{p}$ and $K_{v}$ are position and velocity feedback gains. And, $u^{j}$ is the power amplifier current input.

\section{Constraint Checking and Safety Assurance}

Having to deal with priori unknown maneuvers, telerobotic golf system requires thorough constraint checking of the remote operator position commands. All maneuverability constraints can be categorized into three groups as follows:

(a) Joint limits: All rotary joints have their working ranges. Once the remote operator command is transformed into joint co-ordinates using (1), (2), and (3). Joint positions are checked to verify that they remain within their respective working ranges $\left[\theta_{\text {limit }}^{j}, \theta_{\text {limit }+}^{j}\right] ; j=1,2,3$.

(b) Maximum reach: First joint is a vertical axis joint, thus, can be eliminated from the calculation of tool reach. 
The maximum stretch of the manipulator with respect to the second joint is $L_{2}+L_{3}$. And, if the remote operator command $(x, y, z)$ satisfies

$L 2+L 3-\sqrt{\left(\sqrt{x^{2}+y^{2}}-L_{1}\right)^{2}+z^{2}}<\delta$, then, for any arbitrary command $(x, \bar{y}, z)$, the manipulator is safe from a distance of $\delta$ from the full-stretch singularity. (c) Minimum putter height: The putter should always stay above the ground height $h_{\text {ground }}$. If the constraint $z>h_{\text {ground }}$ is satisfied, it is guaranteed that the putter does not come in contact with the ground.

All these constraints are checked at the remote operator terminal. Once all checks are passed, the command is sent to the local controller. This way, the safe operation of the telerobotic golf game is assured.

\section{Remote Operator Interface}

A keyboard interface was selected as it is the most common computer peripheral, which can be used as a teleoperator interface. This way, the telerobotic game could be played by anyone from anywhere in the world through his keyboard, after logging into the remote operator terminal in KAIST. The keys were assigned as shown in Fig. 3 to be consistent with the motion directions of the putter shown in Fig. 2(a).

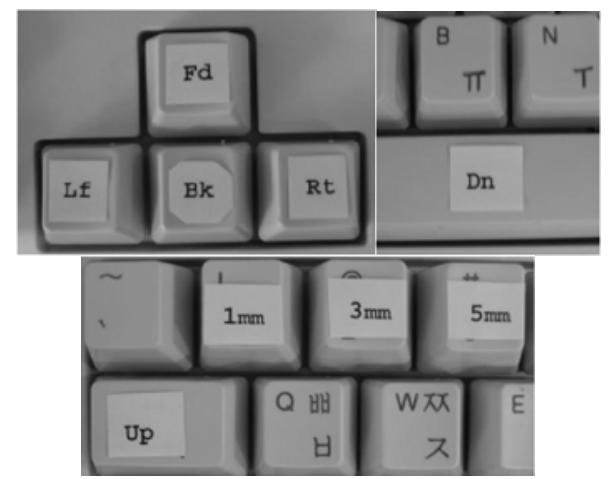

Fig.3 Teleoperator's keyboard configuration

The key assignment is as follows

1) The four arrow keys were assigned to $X-Y$ motion as $\uparrow$ (+X:Forward), $\downarrow$ (-X:Backward), ${ }^{-} \leftarrow$ (+Y:Left) , and $\rightarrow$ (- Y:Right $)$

2) Vertical motion was assigned to "tab" key(+Z:Up), and "space bar"(-Z:Down)

3) 1, 2, and 3 numeric keys were assigned for step lengths of $1[\mathrm{~mm}], 3[\mathrm{~mm}]$, and $5[\mathrm{~mm}]$, respectively.

This key assignment was purely arbitrary, based on intuition. One rationale however is that this configuration is consistent with the natural finger positioning on the standard computer keyboard.
Challenges for the teleoperator

There are few difficulties that challenge the teleoperator stated as follows

1) Putter orientation: Putter is an extension of the prismatic fifth link, and the telerobot is operated using the first three joints. Having no motion at the fourth rotary joint, putter remains fixed at the same joint position relative to the third link. However, to assist the teleoperator, the fourth link is pre-adjusted to an approximate average vertical configuration within the golf-court. The prismatic fifth joint is also not operated, and it avoids the teleoperator rotate the putter the way he prefers.

2) Diagonal motions: Remote operator commands are limited to individual motions along $\mathrm{X}-\mathrm{Y}-\mathrm{Z}$ directions. There is no way to make other movements that combines these individual motions.

3) 2D visual feedback: Visual feedback provides only 2D view of the golf-court at a fixed camera position. It does not provide direct information of the relative putter position. And, no control of the camera positioning is provided.

The teleoperators are expected to experience these challenges and deal with them by interactive learning.

\section{IMPLEMENTATION}

The remote operator terminal was implemented on RedHat Linux 8.0 with kernel version 2.4.20-19.8. The local controller was implemented on Debian GNU Linux 3.0, with real-time kernel 2.4.4-rtl. Due to network security reasons, local controller has not been assigned a global IP address, but it runs a virtual private network that issues a local IP address for the remote operator terminal. The visual feedback was implemented with Microsoft Net-meeting utility, with a single, inexpensive web-camera located at the telerobotic golf-court. Teleoperator's keyboard commands are checked against the working range of joints [-152,152](joint 1), [$45,140]$ (joint 2), and [-142.5,142.5](joint 3) before sending to the local controller. There were eleven players involved from both KAIST and Saga University. They were explained that the operation is safe on their possible wrong commands, and that they are expected to reach the goal as quickly as possible, while learning by mistakes. Their courses of actions were recorded as movie clips, relayed, and evaluated.

\subsection{Evaluation Criterion}

The evaluation criterion was developed on the following measures of errors and skills. There were three recognized errors

1) E1: Hole error: If the player had to significantly change the direction of motion at the close vicinity of the hole, it is judged as a hole error. 

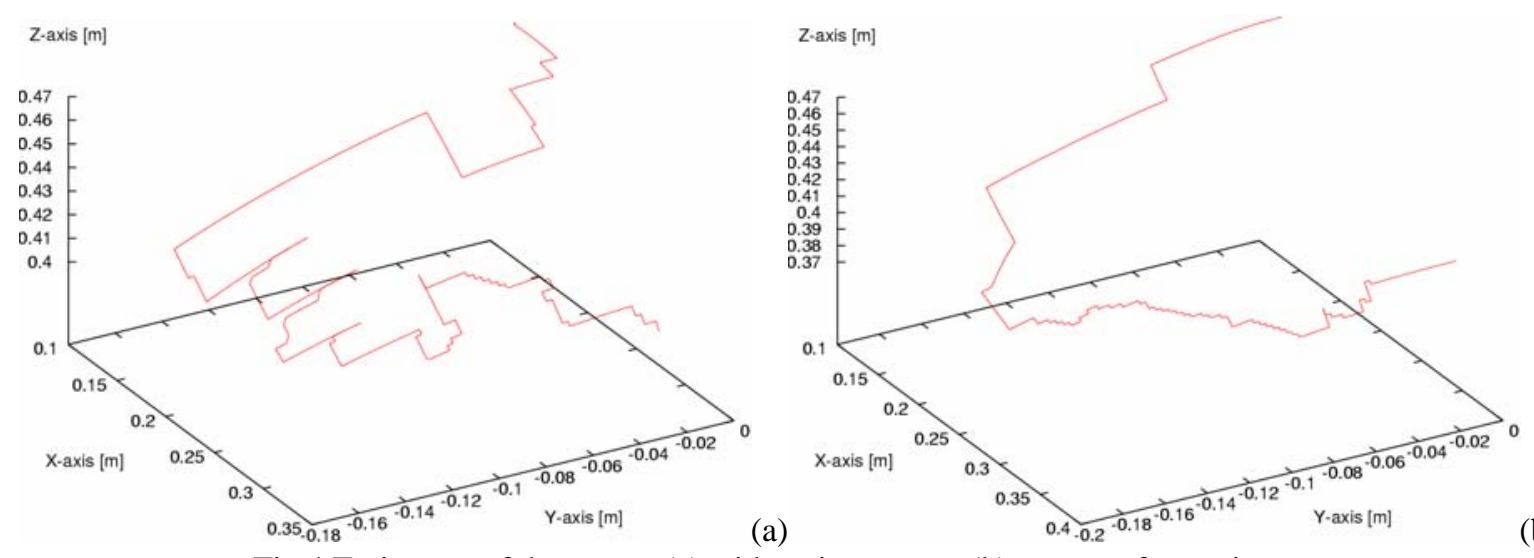

Fig.4 Trajectory of the putter, (a) with serious errors (b) an error-free trajectory

TABLE I-1: Test 1 statistics - for fast keyboard operators

\begin{tabular}{|c|c|c|c|}
\cline { 2 - 4 } \multicolumn{1}{c|}{} & \multicolumn{3}{c|}{ Errors (E1,E2,E3), sk111s (P1, P2), and t1m1ng } \\
\hline User \# & Trial \#1 & Trial \#2 & Trial \#3 \\
\hline K1 & $(0, \mathrm{E} 2+, 0),(0,0),(54)$ & $(0, \mathrm{E} 2-, \mathrm{E} 3),(0,0),(51)$ & $(0,0,0),(\mathrm{P} 1-, 0),(43)$ \\
\hline $\mathrm{K} 3$ & $(0, \mathrm{E} 2, \mathrm{E} 3),(0,0),(109)$ & $(0,0,0),(\mathrm{P} 1-, \mathrm{P} 2-),(36)$ & $(0,0,0),(\mathrm{P} 1+, 0),(45)$ \\
\hline
\end{tabular}

TABLE I-2: Test 1 statistics - for slow keyboard operators

\begin{tabular}{|c|l|l|l|}
\cline { 2 - 4 } \multicolumn{1}{c|}{} & \multicolumn{2}{|c|}{ Errors (E1,E2,E3), sk111s (P1, P2), and t1m1ng } \\
\hline User \# & \multicolumn{1}{|c|}{ Trial \#1 } & \multicolumn{1}{|c|}{ Trial \#2 } & Trial \#3 \\
\hline $\mathrm{K} 2$ & $(0,0,0),(\mathrm{P} 1-, \mathrm{P} 2-),(66)$ & $(0,0, \mathrm{E} 3),(0,0),(75)$ & $(\mathrm{E} 1,0,0),(\mathrm{P} 1-, \mathrm{P}-),(55)$ \\
\hline $\mathrm{K} 4$ & $(0, \mathrm{E} 2-, 0),(0, \mathrm{P} 2),(116)$ & $(0,0,0),(0, \mathrm{P} 2+),(59)$ & $(0,0,0),(\mathrm{P} 1, \mathrm{P} 2-),(52)$ \\
\hline $\mathrm{K} 5$ & $(\mathrm{E} 1, \mathrm{E} 2+, \mathrm{E} 3+),(0, \mathrm{P} 2),(105)$ & $(0, \mathrm{E} 2,0),(0, \mathrm{P} 2),(68)$ & $(0, \mathrm{E} 2-, 0),(0, \mathrm{P} 2),(59)$ \\
\hline $\mathrm{K} 6$ & $(\mathrm{E} 1, \mathrm{E} 2+, \mathrm{E} 3),(0,0),(103)$ & $(0, \mathrm{E} 2, \mathrm{E} 3),(0,0),(51)$ & $(0, \mathrm{E} 2-, \mathrm{E} 3),(0,0),(49)$ \\
\hline $\mathrm{K} 7$ & $(\mathrm{E} 1, \mathrm{E} 2+, \mathrm{E} 3),(0,0),(101)$ & $(0,0,0),(\mathrm{P} 1-, \mathrm{P} 2-),(57)$ & $(0,0, \mathrm{E} 3),(\mathrm{P} 1-, \mathrm{P} 2-),(71)$ \\
\hline
\end{tabular}

2) E2: Landing error. If the player had to make many moves off the ground before landing the putter behind the ball, it is judged as a landing error.

3) E3: Steering error: If the player failed to keep putting the ball from behind, it is judged as a steering error

And, there were two recognized skills

1) P1: Landing skill. If the player positioned the stick behind the ball without going through many moves off the ground, it is judged as a landing skill.

2) P2: Steering skill. If the player could keep steer the ball towards the hole, without going through other directions, it is judged as a steering skill.

\subsection{Tests}

Test 1: The first test aimed to investigate the interactive learning of human teleoperators, and reveal how they learn to enhance the performance.
This test was carried out by seven players at KAIST(Korea). Each of them were given three trials starting with the same configuration as shown in Fig. 2(a). Figure 4 shows two trials in which the errors can be visualized and compared with an error-free trial.

Clearly, there are two categories of keyboard operators; fast operators and slow operators. The teleoperation characteristics significantly depend on this issue, and therefore, the performance were separately categorized in TABLE I-1, and TABLE I-2. Errors and skills are further evaluated and tagged as "-"(slight), “no sign"(considerable), and " +"(serious). This grading was carried out by inspection of the movie clips. Errors and skills were further converted to numerical form by the following marking scheme:

1) slight errors or skills $=1$ points

2) considerable errors or skills $=2$ points

3) serious errors or skills $=3$ points 

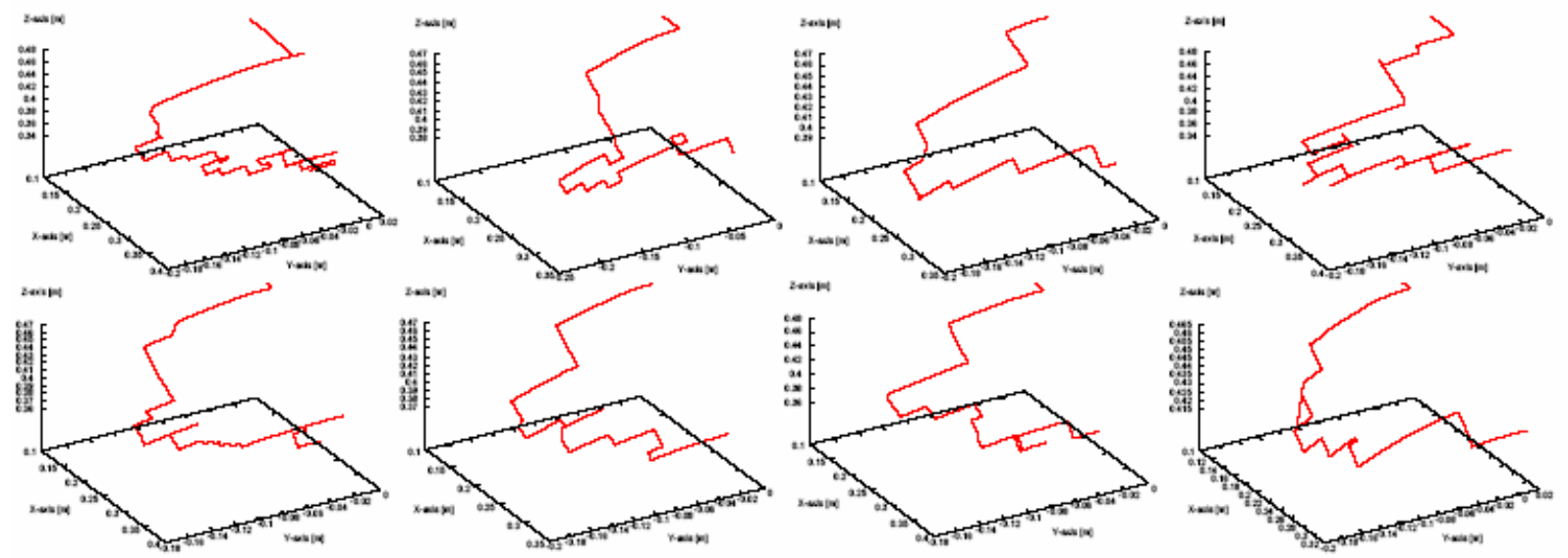

Fig.5 Trajectories at the visual feedback switching: Top - last trial with naked-eye visual feedback, Bottom - first trial with camera feedback

TABLE II: Test 2 statistics

Naked-eye visual feedback

\begin{tabular}{|c|c|c|c|}
\hline & \multicolumn{3}{|c|}{ Errors $(E 1, B 2, B 3), \operatorname{sk} 111 \mathrm{~s}(\mathrm{P} 1, \mathrm{P} 2)$, and t1m1ng $(t)$} \\
\hline User \# & Tr1al \#1 & Tr1al \#2 & Trial \#3 \\
\hline s1 & $\left(E_{1}, E_{2}, E_{3}\right),(0,0),(80)$ & $\left(0,0, \mathrm{E}_{3}\right),(\mathrm{P} 1,0),(37)$ & $(\mathrm{E} 1,0, \mathrm{E} 3+),(\mathrm{P} 1-, 0),(86)$ \\
\hline $\mathrm{s}_{2}$ & $\left(0, E_{2}+, E_{3}+\right),(0,0),(73)$ & $\left(0,0, \mathrm{E}_{3}\right),(\mathrm{P} 1,0),(47)$ & $(0,0, \mathrm{E} 3),(\mathrm{P} 1-, 0),(50)$ \\
\hline s3 & $\left(E_{1}, E_{2}, E_{3}\right),(0,0),\langle 78\rangle$ & $\left\langle\mathrm{E}_{1}, 0, \mathrm{E} 3\right),\langle\mathrm{P} 1-, 0\rangle,\langle 46\rangle$ & $(\mathrm{E} 1,0, \mathrm{E} 3-\rangle,(\mathrm{P} 1+, 0),(36)$ \\
\hline s4 & $\left(\mathrm{E}_{1}, \mathrm{E}_{2}, \mathrm{E}_{3}+\right),(0,0),(86)$ & $\left\langle 0, E_{2}+, E_{3}+\right),\langle 0,0\rangle,\langle 60\rangle$ & $(0, \mathrm{E} 2+, \mathrm{E} 3+),(0,0),(65)$ \\
\hline
\end{tabular}

Camera visual feedback

\begin{tabular}{|c|c|c|c|}
\hline & Trial \#4 & Tr1al \#5 & Trial \#6 \\
\hline s1 & $\left(\mathrm{E}_{1}, 0, \mathrm{E}_{3}\right),(\mathrm{P} 1-, 0),(90)$ & $(\mathrm{E} 1,0,0),(\mathrm{P} 1-, \mathrm{P} 2-),(80)$ & $\langle 0,0,0\rangle,(\mathrm{P} 1-, \mathrm{P} 2-),\langle 80\rangle$ \\
\hline s2 & $(0,0, E 3),\langle\mathrm{P} 1-, 0\rangle,\langle 61\rangle$ & $\langle 0,0, \mathrm{E} 3-),(\mathrm{P} 1+, 0),\langle 37\rangle$ & $\langle 0,0,0\rangle,(\mathrm{P} 1+, \mathrm{P} 2-),\langle 36\rangle$ \\
\hline s3 & $\left(0,0, E_{3}\right),\langle\mathrm{P} 1-, 0),\langle 97\rangle$ & $(0,0, \mathrm{E} 3),(\mathrm{P} 1-, \mathrm{P} 2),(63)$ & $\langle 0,0,0\rangle,(\mathrm{P} 1-, \mathrm{P} 2),\langle 58\rangle$ \\
\hline S4 & $\left(0, E_{2}-, E_{3}+\right),(0,0),(71)$ & $\langle 0, E 2-, E 3+),\langle 0,0\rangle,\langle 81\rangle$ & $(0,0, E 3\rangle,(\mathrm{P} 1-, 0),\langle 52\rangle$ \\
\hline
\end{tabular}

Then, the error and skill counts were accumulated and graphed in Fig. 6(a).

Test 2: The second test aimed to investigate the key issues of the camera vision problem and how it affects the remote operator interaction and the entire performance of the telerobotic system. This test was carried out by four players at Saga University(Japan). These players made telnet-login to the remote operator terminal at KAIST, and performed six trials each. The first three trials of each player were carried out with naked-eye visual feedback. In order to get a consistent visual feedback, players positioned at the camera position in these trials. In their last three trials, they teleoperated the robot with the camera visual feedback. Figure 5 shows the trajectories of all four players at the time of visual feedback switching from naked-eye to camera. The performance is listed in TABLE II. The error and skill counts for the two tests are graphed in Fig. 6(b) and Fig. 6(c).

\subsection{Results}

Test 1:

The results in TABLE $\mathrm{I}-1$, and TABLE $\mathrm{I}-2$ can be interpreted as follows:

1) Fast-player K1, first tries to exploit (avoid errors) and then explore (exercise new skills). He recorded consistent improvement in successive trials. He explored landing skills (P1) and ended up with much better timing of 43 [s]. He seems to be an already learned player (by other means of computer games). The fast-player K3, attempts exploitation and exploration simultaneously, taking a lot of risks. Not taking time to learn caused lot 

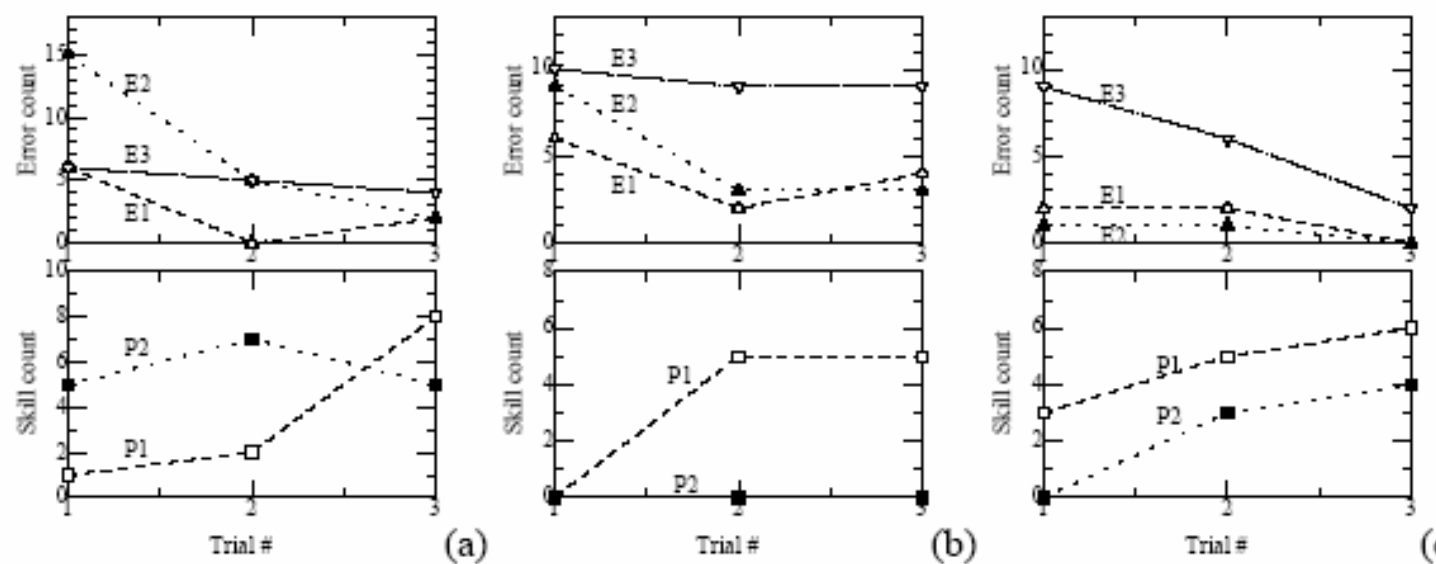

Fig.6 Error and skill counts: (a) Test 1, (b) Test 2: with naked-eye vision, and (c) Test 2: with camera vision

of errors in his very first trial. However, he has been extremely successful in the second trial both in landing (P1) and steering (P2) recording the overall minimum timing of 36[s]. His learning and adaptation is significantly fast.

2) The slow-players K4, K5, K6, and K7 have shown highly comparable and consistent statistics. Though their cautious behavior cost a significantly long time in the first trial due to going through all kinds of errors (E1, E2, E3). However, they are capable of quickly reducing hole error (E1) in the second trial onwards. They are inherently slow interactive, and records their best timing longer than that of the fastplayers.

3) Unlike other slow-player, K7 shows exploring skills (P2) in his third trial, and cost additional time as it was not successful.

4) Slow-payer K2 shows somewhat already learned behavior comparable to the second trial of other slowplayers.

Figure 6(a) verifies that the players learn to avoid errors very quickly, and that they learn new skills to properly operate the telerobot.

\section{Test 2:}

The results in Fig. 6(b) and Fig. 6(c) clearly show that there is no significant discontinuity of error and skill learning profiles due to switching from naked-eye vision to camera vision. The experience with the naked-eye vision is carried into the successive trial with the camera vision. This indicates to be an effective training procedure for human teleoperators in actual applications.

\section{DISCUSSION}

Following observations have been made during the trials.
1) Although the two-dimensional camera vision does not provide adequate feedback information for the teleoperator, he is capable of learning quickly by experience and guess-work. Practice with naked-eye vision significantly helps human teleoperator to accurately conceive the work-site geometry and also to exercise predictive control actions when he deals with camera vision.

2) Camera vision can be supplemented with a laser pointer attached to the putter, and beaming downward. The beam indicates the projection of the putter on the X$\mathrm{Y}$ plane, which will be a useful guide for the teleoperator. 3) The round trip transmission delay was measured by a "ping-test" as $14[\mathrm{~ms}] / 20[\mathrm{~ms}] / 28[\mathrm{~ms}]$ in the minimum/average/mean deviation format. Visual feedback adds more time to these statistics. This delay has been slightly noticed by almost all players. Yet, they did not experience any difficulty in practicing their predictive control actions.

4) Predictive control is attributed to teleoperation. As predictive control is based on the velocity of motion, it is necessary to maintain a continuous motion to exploit cognitive prediction skills of the teleoperator. It was clearly observed that many operators prefer to use small step lengths $(1[\mathrm{~mm}]-3[\mathrm{~mm}])$ and keep issuing commands and maintain a velocity throughout the operation. On the other hand, they dislike move-and-wait mode of control as waiting significantly retards their predictive control capabilities.

5) Key configuration of the keyboard should comply with the telerobot's motion directions. Using arrow keys for $\mathrm{X}-\mathrm{Y}$ motion is effective, whereas "tab" and "space bar" are not. For $\mathrm{Z}$ axis motions, a better configuration would be "A" and " $\mathrm{Z}$ " alphabet keys, which are the same keys that are used in most computer games.

6) More crucial factor that consumes time is wrong commands due to wrong key strokes. All players demonstrated this confused interaction with the keyboard intermittently. However, with more appropriate key 
assignment, and also with practice, this problem can be completely solved.

7) Without being able to perceive the height of the "putter" from the camera view in Fig. 2(a), there was a slight tendency that the ball being putted too high off the ground, violating the condition $h<r$ in Fig. 2(c). It produces an excessive force $R=w+f_{v}$ on the ground, that would be disastrous. To avoid this, either camera angle could be adjusted, or a local force feedback could be implemented.

8) To further improve controllability, fourth link can also be synchronously controlled with the second and third joints in order to compensate the deflection of putter orientation due to the motions of other rotary joints. This way, the putter will stay vertical, giving a better response for the teleoperator.

9) Task oriented camera position control would be an effective way of exploiting the vision information. It would also be an effective alternative to sophisticated graphics-based techniques.

\section{CONCLUSION}

Human teleoperators learn and adapt very quickly to a properly designed teleoperator interfaces. A very few trials is enough for them to learn how to avoid errors and learn new skills even without sophisticated graphics-based interfaces. A general learning pattern is to exploit error avoidance first, and then explores new skills despite the delay and vision problems. A significant fraction of teleoperators show a very steady and consistent learning pattern. Although twodimensional camera visual feedback significantly affects teleoperator performance, training with nakedeye vision can significantly help them to exercise accurate teleoperations. This could be a very effective training procedure for human teleoperators before they are deployed for actual teleoperations. Keyboard interface has shown to be very effective and easy to learn with, as it provides the operator a quantitative measure about his own commands. It helps him to predict the movements of the telerobot more accurately. To further help the prediction accuracy, small step sizes and a more continuous motion are useful. Simple techniques such as pointed beams (laser) and task-oriented camera position control can be incorporated to help the teleoperator with vision problems.

\section{REFERENCES}

[1] G. Hirzinger, B. Brunner, J. Dietrich, and J. Heindl, "Sensor-based space robotics-ROTEX and its telerobotic features," IEEE Tr. Robot. and Automat.\}, vol. 9, no. 5, pp. 649-663, 1993.

[2] P. G. Neckes and M. K. Long, "Local-remote telerobotics for underwater vehicles," Proc. Symp. on
Autonomous Underwater Vehicle Technology\}, pp. 1115, 1992.

[3] A. Rovetta, R. Sala, W. Xia, and A. Togno, “Remote control in telerobotic surgery," IEEE Tr. Syst., Man and Cyber. Part A\}, vol. 26, no. 4, pp. 438-444, 1996.

[4] S. R. Munasinghe and M. Nakamura, “'Teleoperation of welfare robotic systems by motion planning considering assigned velocity and acceleration limit," Intl. J. of Human-Friendly Welfare Robotic Systems, vol. 3, no. 2, pp. 23-31, 2002.

[5] A. Rovetta, "FRIEND robot, space telerobot for rescue and recovery of astronauts," IEE/RSJ International Workshop on Intelligent Robots and Systems, vol. 3, pp. 1663-1668, 1991.

[6] K. Goldberg, "'Mercury Project: A feasibility study for internet robots," IEEE Robotics and Automation Magazine, pp. 35-40, March 2000.

[7] K. Taylor, "Internet robotics: A new robotics niche," IEEE Robot. and Automat. Magazine, pp. 27-34, March 2000.

[8] K. Goldberg, ' $T$ The robot in the garden, Telerobotics and telepistemology in the age of the Internet”, MIT Press, Cam, Mass: 2000.

[9] T. B. Sheridan, "Telerobotics, Automation, and Human Supervisory Control”, MIT Press, Cam, Mass, 1992.

[10] T. B. Sheridan, ' Space teleoperation through time delay," IEEE Tr. Robot., and Automat., vol. 9, no. 5, pp. 592-605, 1993.

[11] E. Freund, and J. Rossmann, 'Projective virtual reality: Bridging the gap between virtual reality and robtics," IEEE Tr. Robot., and Automat., vol. 15, no. 3, pp. 411-422, 1999.

[12] A. Liu, G. Tharp, L. Fench, S. Lai, and L. Stark, "Some of what one needs to know about using headmounted displays to improve teleoperator performance," IEEE Tr. Robot., and Automat., vol. 9, no. 5, pp. 638-648, 1993.

[13] J. E. Loyd, J. S. Bies, D. K. Pai, and D. G. Lowe, "Model-based telerobotics with vision," IEEE Intl Conf. on Robot. and Automat., pp. 1297-1304, April, 1997.

[14] M. Sonka, V. Hlavac, and R. Boyle 'Image Procesing, Analysis, and Machine Vision, 2nd ed. Pacific Grove CA: PWS, ITP Inc., 1999.

[15] E. P. Kan, 'Man-machine interface of a teleroboic system," Intl. Conf. on Intel, Robots and Syst., pp. 564572, Sep., 1989. 